Document downloaded from:

http://hdl.handle.net/10251/60806

This paper must be cited as:

Limones Herrero, D.; Pérez Ruiz, R.; Jiménez Molero, MC.; Miranda Alonso, MÁ. (2014).

Retarded photooxidation of cyamemazine in biomimetic microenvironments. Photochemistry and Photobiology. 90(5):1012-1016.

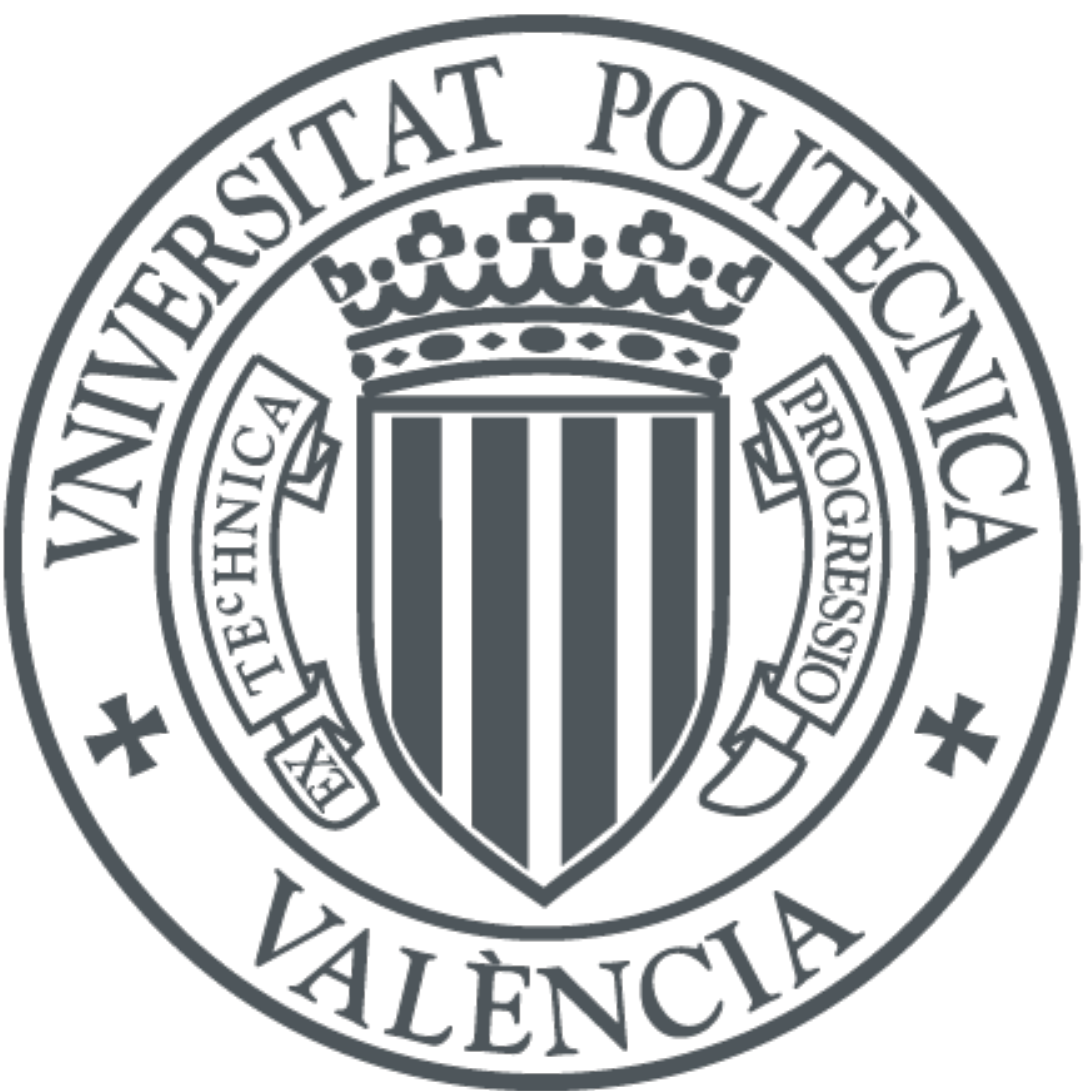

The final publication is available at

http://dx.doi.org/10.1111/php.12303

Copyright Wiley

Additional Information 


\title{
Retarded Photooxidation of Cyamemazine in Biomimetic Microenvironments
}

\author{
Daniel Limones-Herrero, Raúl Pérez-Ruiz, M. Consuelo Jiménez* and Miguel A. \\ Miranda* \\ ${ }^{a}$ Departamento de Química-Instituto de Tecnología Química UPV-CSIC, Universitat \\ Politècnica de València, Camino de Vera s/n, Valencia, Spain. mcjimene@quim.upv.es; \\ mmiranda@qim.upv.es
}

\section{Introduction}

The phenothiazine (PTZ) tricyclic ring system is incorporated into a number of drugs that cover a broad spectrum of pathologies. Members of this family display a wide range of biological activities and are commonly used as antipsychotic, tranquilizing or antihistaminic agents. ${ }^{1,2,3,4}$

Due to the fact that PTZ is an active chromophore, the photochemical behavior of PTZ-containing drugs has attracted considerable attention, in connection with the possible photosensitizing side-effects experienced by treated patients. ${ }^{5,6,7}$ In this context, it has been described that the key photophysical parameters of PTZs can be influenced by factors such as the substitution at positions 1 and 2 , the nature of the solvent or the excitation energy. ${ }^{8,9,10}$

Transport proteins present in blood and plasma bind and carry a vast array of pharmaceuticals and consequently are important modulators of pharmacokinetics and disposition. The most abundant are serum albumins (SAs) and $\alpha_{1}$-acid glycoproteins (AAGs). ${ }^{11,12}$ The interaction of several PTZ neuroleptics with AAGs has been investigated using circular dichroism and equilibrium dialysis techniques. In the case of human AAG (HAAG) it has been concluded that PTZ derivatives bind preferentially to 
only one common binding site, where hydrophobic forces are mainly involved, although electrostatic interactions also participate. ${ }^{13,14,15}$ The affinity of PTZs for HAAG has been employed for the development of glycoprotein-based stationary phases for the resolution of enantiomers by HPLC. ${ }^{16,17,18}$

Effective inclusion of PTZs within other biomimetic systems, such as $\beta$ - or $\gamma$ cyclodextrins (CDXs) or sodium dodecyl sulfate (SDS) micelles has also been observed. ${ }^{19,20,21}$ With CDXs, PTZs inclusion complexes with different stoichiometries are formed. Moreover, encapsulation within $\beta$-CDX protects human erythrocytes from photohemolysis induced by a number of PTZs. ${ }^{22}$ Enantioseparations of chiral PTZs using various CDXs as chiral selectors has been exploited for the development of analytical applications. ${ }^{23}$

Inclusion of PTZs within sodium dodecyl sulfate (SDS) micelles has been investigated using both absorption and fluorescence spectroscopies, looking at the influence of the PTZs protonation state on the interaction and at the competition between photoionization and triplet excited state formation.

Cyamemazine (CMZ, 2-cyano-10-(3-dimethylamino-2-methylpropyl)phenothiazine, Chart 1) is a PTZ derivative, employed as neuroleptic drug, which exhibits strong cutaneous phototoxicity in humans. ${ }^{24}$ The drug is readily photooxidized upon UVA irradiation to 2-cyano-10-(3-dimethylamino-2-methylpropyl)phenothiazine $N, S$-dioxide (CMZ-SONO), which is in turn an effective photodynamic agent able to produce singlet oxygen. This photoproduct is also formed during UVA irradiation of cultured human fibroblasts incubated with the drug. Since CMZ is mainly localized in the lysosomes of human fibroblasts and keratinocytes, CMZ-SONO plays a central role in the development of phototoxic responses in humans treated with CMZ. ${ }^{25,26}$ 
The CMZ fluorescence spectrum in phosphate buffered solution (PBS, $\mathrm{pH}=7.4$ ) displays a maximum at $c a .530 \mathrm{~nm}$, and the fluorescence quantum yield is 0.11 at $\lambda_{\text {exc }}=$ $267 \mathrm{~nm}$. Laser flash photolysis (LFP) under the same conditions shows the transient spectrum of the triplet excited state $\left({ }^{3} \mathrm{CMZ}^{*}\right)$, with a characteristic band at $c a .420 \mathrm{~nm}$, together with the corresponding radical cation $\left(\mathrm{CMZ}^{+\bullet}\right)$ absorbing strongly in the 500 $\mathrm{nm}$ region. Both species react with oxygen, although with markedly different rate constants $\left(4 \times 10^{9}\right.$ and $2 \times 10^{7} \mathrm{M}^{-1} \mathrm{~s}^{-1}$, respectively).<smiles>CC(CN(C)C)CN1c2ccccc2Sc2ccc(C#N)cc21</smiles>

CMZ<smiles>CC(CN1c2ccccc2S(=O)c2ccc(C#N)cc21)C[N+](C)(C)[O-]</smiles>

CMZ-SONO

Chart 1. Chemical structures of CMZ and its photoproduct CMZ-SONO.

A number of relevant processes occur in well-defined biological compartments; hence, the behavior of drugs within biomimetic microenvironments is an important issue. In this work, the photobehavior of enantiomerically pure CMZ has been examined within AAGs, $\beta-$ and $\gamma-\mathrm{CDX}$, as well as SDS. The results show that phootoxidation is markedly retarded in SDS and AAGs, where the local hydrophobicity prevents $\mathrm{CMZ}$ photoionization.

\section{Experimental Section}

Materials and Solvents. HAAG, BAAG, $\beta$-CDX, $\gamma$-CDX and SDS were commercially available. Spectroscopic and HPLC-grade solvents were used without further 
purification. Solutions in PBS $(0.01 \mathrm{M}, \mathrm{pH}=7.4)$ were prepared by dissolving the commercial tablets in deionized water.

Racemic CMZ tartrate was kindly provided by Aventis (Vitry-sur-Seine, France) and kept in the dark at $4{ }^{\circ} \mathrm{C}$. The free base was obtained by liquid-liquid extraction of $\mathrm{CMZ}$ tartrate, using $1 \mathrm{M} \mathrm{NaOH} / \mathrm{CH}_{2} \mathrm{Cl}_{2}$. The organic phase was dried with $\mathrm{Na}_{2} \mathrm{SO}_{4}$, filtered and evaporated in vacuo to afford racemic CMZ. Pure (-)-CMZ and (+)-CMZ were isolated from the racemic mixture by HPLC (see Supporting Information, pp S2S7 for experimental details and NMR characterization).

Fluorescence experiments. Solutions of (-)- or (+)-CMZ in the absence and presence of different amounts of guests were placed in $10 \times 10 \mathrm{~mm}^{2}$ quartz cells. The absorbance at the excitation wavelength $(330 \mathrm{~nm})$ was kept below 0.2. Experiments were performed at $22{ }^{\circ} \mathrm{C}$. Emission spectra were recorded on a JASCO FP-8500 spectrofluorometer system, provided with a monochromator in the wavelength range of 200-850 nm. Time-resolved measurements were performed with a TimeMaster fluorescence lifetime spectrometer TM-2/2003 from PTI by means of the stroboscopic technique, which is a variation of the boxcar technique. A hydrogen/nitrogen flashlamp was used as excitation source.

Laser flash photolysis measurements. Experiments were carried out with a pulsed Nd:YAG Laser system with $355 \mathrm{~nm}$ as excitation wavelength, $0.6 \mathrm{~cm}$ as beam diameter and a pulse duration of $10 \mathrm{~ns}$. The energy was set at $15 \mathrm{~mJ} / \mathrm{pulse}$. The apparatus consisted of the pulsed laser, a xenon lamp, a monochromator, and a photomultiplier. Samples of (-)- or (+)-CMZ in the absence and presence of different amounts of guests were placed in $10 \mathrm{~mm} \times 10 \mathrm{~mm}$ quartz cells. The absorbance at the excitation wavelength was kept at 0.2 . Experiments were performed at $22{ }^{\circ} \mathrm{C}$. In general, samples received 2 pulses for all of the kinetic measurements. This light dose did not result in 
any detectable decomposition of the sample, as revealed by UV-Vis absorption prior to and after photolysis.

Steady-state irradiations. Solutions of (-)- or (+)-CMZ $6 \times 10^{-5} \mathrm{M}$ in PBS/air were placed into quartz cells and irradiated at different times with monochromatic light from a $150 \mathrm{~W}$ Xenon lamp at $\lambda_{\text {exc }}=355 \mathrm{~nm}$. The course of the reactions was followed by recording the changes in the emission spectra $\left(\lambda_{\mathrm{exc}}=330 \mathrm{~nm}\right)$.

\section{Results and Discussion}

The two enantiomers (-)- and (+)-CMZ were isolated from the commercial racemic drug, using chromatographic (HPLC) resolution with a chiral stationary phase (Figure S1). As expected, the absorption spectra of both compounds in PBS were identical, and displayed an intense band with maximum at $270 \mathrm{~nm}$ and a tail reaching $400 \mathrm{~nm}$ (Figure S2). Excitation was performed at this longer-wavelength tail, to avoid interferences due to light absorption by the host.

Fluorescence studies. Selective excitation of $\mathrm{CMZ}$ in the presence of AAGs was achieved at $330 \mathrm{~nm}$. Fluorescence measurements were used to follow formation of CMZ@AAG complexes, through the observed changes in the position and intensity of the emission band, as well as in the decay traces. The results obtained for (-)-CMZ are shown in Figure 1. In the presence of increasing amounts of AAGs, the emission band of (-)-CMZ (initially peaking at $525 \mathrm{~nm}$ in PBS), underwent a progressive enhancement with a concomitant hypsochromic shift. At 1:2 drug/HAAG molar ratio (Figure 1A), the maximum was $c a .30 \mathrm{~nm}$ blue-shifted, and the quantum yield $\left(\phi_{\mathrm{F}}\right)$ increased from 0.064 (in PBS) up to 0.109 (Table 1). A similar behavior was observed in the presence of the bovine protein BAAG (Figure 1B). This indicated efficient encapsulation of CMZ 
within both AAGs. Accordingly, the fluorescence lifetimes $\left(\tau_{\mathrm{F}}\right)$ were higher for CMZ@AAG complexes than for CMZ in the bulk solution (Table 1).
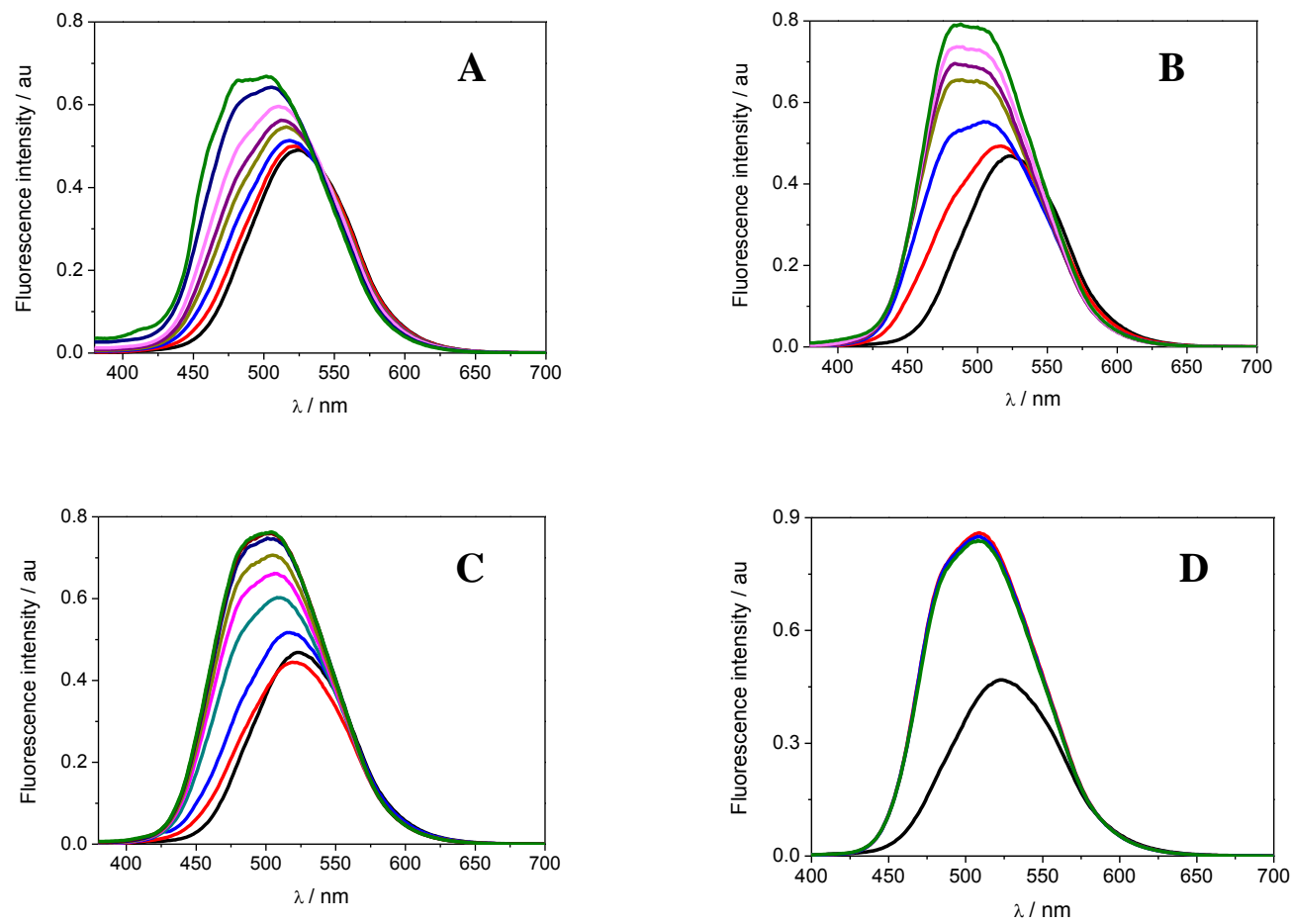

Figure 1. Fluorescence spectra of $(-)-\operatorname{CMZ}\left(6 \times 10^{-5} \mathrm{M}, \lambda_{\mathrm{exc}}=330 \mathrm{~nm}\right.$, PBS/air $)$ in the presence of increasing amounts of A: HAAG (0 to 3 eq. $), \mathbf{B}$ : BAAG (0 to 2 eq. $), \mathbf{C}: \beta$ CDX (0 to 200 eq.), D: SDS (0 to $\left.1.2 \times 10^{-2} \mathrm{M}\right)$. The corresponding data for $(+)-\mathrm{CMZ}$ are presented in Figure S4 of SI.

As alternative anisotropic media, cyclodextrins ( $\beta$-CDX and $\gamma$-CDX) and anionic micelles (SDS) were selected to investigate the emission behavior of encapsulated CMZ. In general, as mentioned above for AAGs, inclusion of (-)-CMZ within these biomimetic michroheterogeneous systems was associated with a significant blue-shift in the emission maxima and a marked enhancement of the fluorescence intensities (Figures 1C, D and S3). A parallel increase in the values of the related photophysical parameters $\left(\phi_{\mathrm{F}}, \tau_{\mathrm{F}}\right)$ was observed (Table 1$)$. 
Table 1. Fluorescence data for (-)-CMZ in different media.

\begin{tabular}{lccccc}
\hline Host & $\lambda_{\max \text { em }}(\mathrm{nm})$ & $\phi_{\mathrm{F}}$ & $\tau_{\mathrm{F}}(\mathrm{ns})$ & $\mathrm{k}_{\mathrm{F}} \times 10^{7}\left(\mathrm{~s}^{-1}\right)$ & $\mathrm{E}_{\mathrm{s}}(\mathrm{kcal} / \mathrm{mol})$ \\
\hline None & 525 & 0.064 & 5.56 & 1.15 & 67.4 \\
$\mathrm{HAAG}^{\mathrm{a}}$ & $482-505$ & 0.109 & 6.68 & 1.63 & 67.6 \\
$\mathrm{BAAG}^{\mathrm{b}}$ & $483-508$ & 0.118 & 7.93 & 1.49 & 66.8 \\
$\beta-\mathrm{CDX}^{\mathrm{c}}$ & $486-512$ & 0.116 & 7.58 & 1.53 & 67.3 \\
$\gamma-\mathrm{CDX}^{\mathrm{c}}$ & $508-522$ & 0.085 & 6.79 & 1.25 & 67.1 \\
$\mathrm{SDS}^{\mathrm{c}}$ & $496-518$ & 0.117 & 8.76 & 1.33 & 66.2 \\
\hline
\end{tabular}

Drug/guest molar ratio: ${ }^{\mathrm{a}} 1: 3,{ }^{\mathrm{o}} 1: 2,{ }^{\mathrm{c}} 1: 200$

Steady-state photochemistry. After investigating the inclusion of CMZ within the different hosts, the entrapped drug was irradiated under air with monochromatic light $\left(\lambda_{\text {irr }}=355 \mathrm{~nm}\right)$. The course of the reaction was monitored by recording the fluorescence changes of the photomixtures $\left(\lambda_{\mathrm{exc}}=330 \mathrm{~nm}\right)$ at increasing exposure times. Formation of the known photoxidation product (CMZ-SONO, Chart 1) was assessed by the appearance of a new emission band at shorter wavelengths $\left(\lambda_{\max }=420 \mathrm{~nm}\right)$. Figures 2A-D show the results obtained for (-)-CMZ. Interestingly, in the presence of HAAG (Figure 2B) and BAAG (Figure S5A in SI) the reaction was clearly slower than in bulk PBS (Figure 2A). The protective effect of $\beta$ - (Figure 2C) and $\gamma$-CDX (Figure S5B in SI), although detectable, was less marked. The lowest photoreactivity was found inside SDS micelles (Figure 2D). 

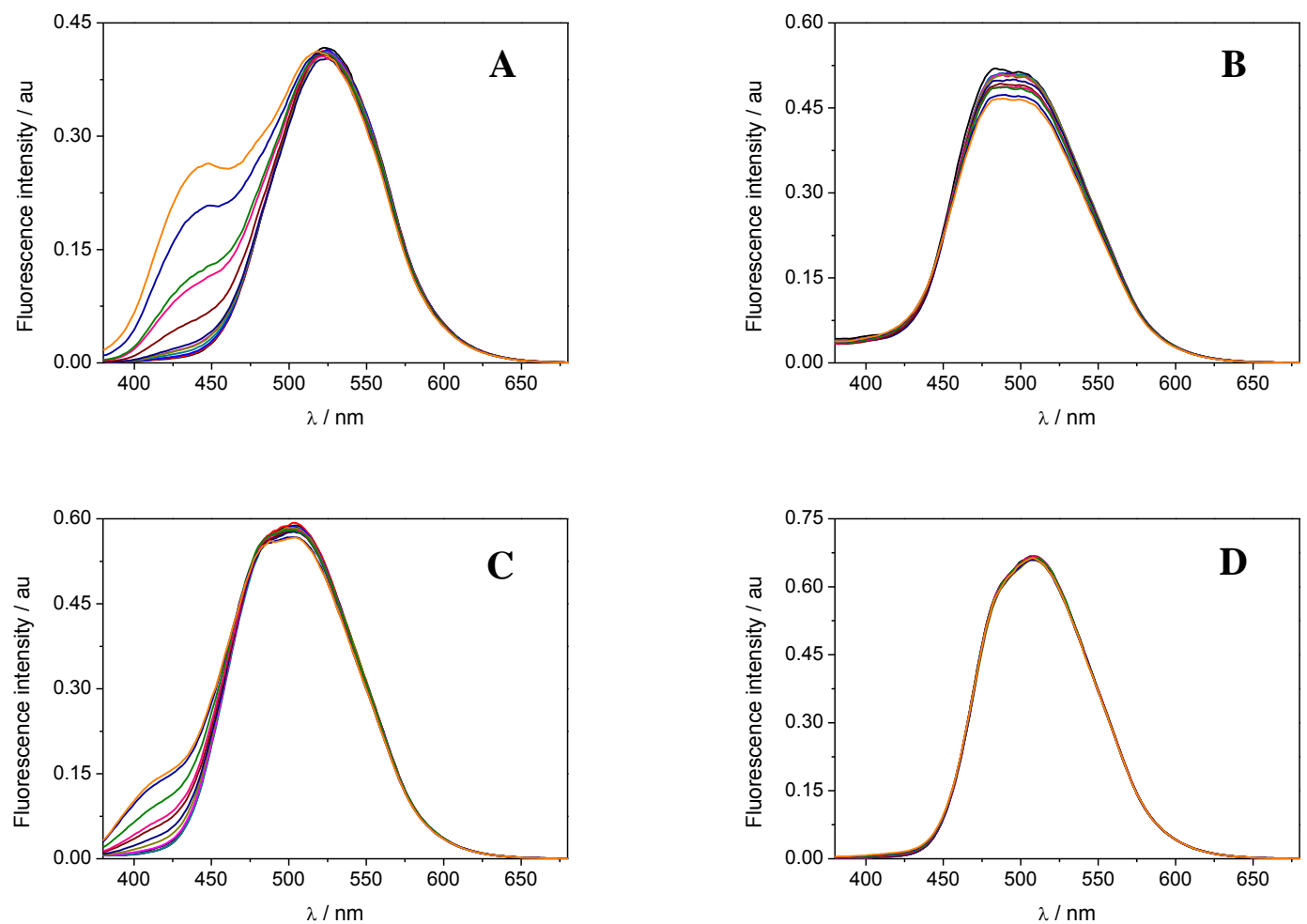

Figure 2. Fluorescence spectra obtained after $355 \mathrm{~nm}$ irradiation of $(-)-\mathrm{CMZ}\left(6 \times 10^{-5}\right.$ $\mathrm{M}, \lambda_{\mathrm{exc}}=330 \mathrm{~nm}$, PBS/air, 0 to $20 \mathrm{~min}$ ) in different media. A: No host. B: CMZ/HAAG 1:3 molar ratio. C: CMZ/ $\beta$-CDX 1:200 molar ratio. D: CMZ/SDS 1:200 molar ratio. The corresponding data for (+)-CMZ are presented in Figure S6 of the SI.

The progress of the reaction at different irradiation times is represented in Figure 3. In all the microheterogeous media the process was much slower than in aqueous solution, with the lowest conversion in SDS.

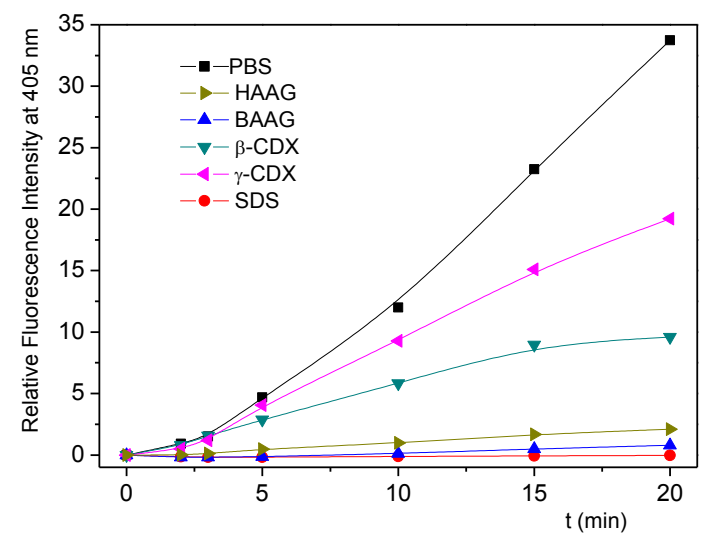

Figure 3. Photooxidation of (-)-CMZ in different media. 
Laser flash photolysis studies. Transient absorption spectroscopy was employed to investigate the involvement of short-lived species in the formation of the photoproduct. Thus, LFP of (-)-CMZ ( $\lambda_{\mathrm{exc}}=355 \mathrm{~nm}, \mathrm{PBS} /$ air $)$ was performed in the different media. In PBS, absorption bands at $320 \mathrm{~nm}\left({ }^{3} \mathrm{CMZ}^{*}\right)$ as well as at $290 \mathrm{~nm}$ $\left(\mathrm{CMZ}^{+}\right)$were observed. In the $400-550 \mathrm{~nm}$ region, the spectra of both species overlap. Remarkably, upon addition of sufficient HAAG or BAAG, the contribution of $\mathrm{CMZ}^{+\bullet}$ became nearly negligible in the $250-350$ region, where ${ }^{3} \mathrm{CMZ}^{*}$ dominated the spectra (Figure 4A). In aerated atmosphere the intraprotein triplet lifetimes were considerably longer than in solution (Figure 4B). This effect was also observed in CDXs, although to a much lower extent.
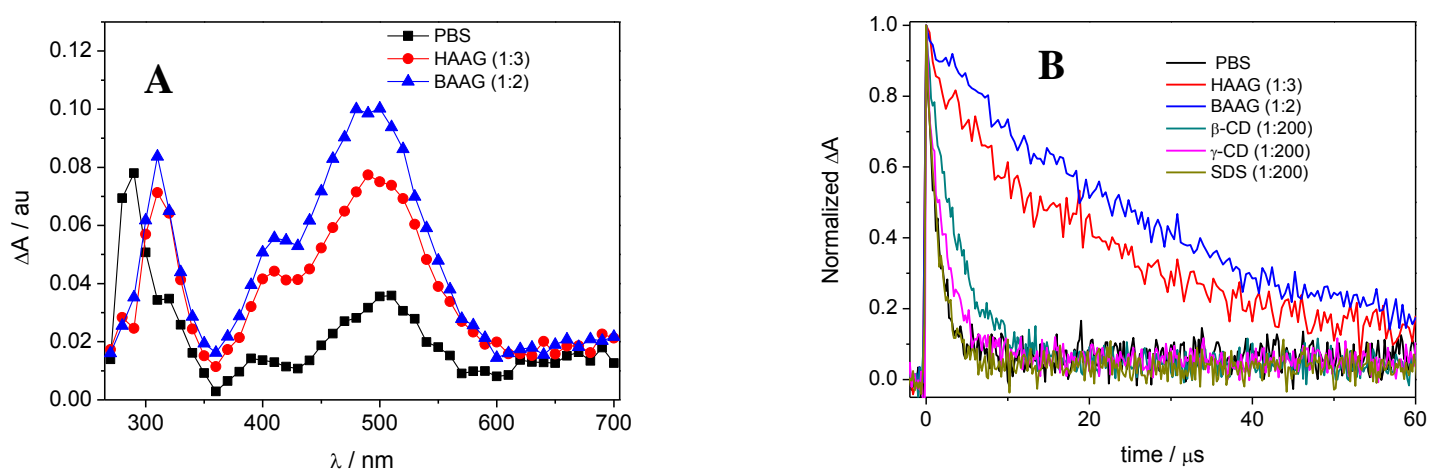

Figure 4. Laser flash photolysis of $(-)-\mathrm{CMZ}\left(\lambda_{\mathrm{exc}}=355 \mathrm{~nm}\right.$, PBS/air) alone and in the presence of two equivalents of AAGs. A: transient absorption obtained $0.5 \mu$ s after the laser pulse. B: Decay traces monitored at $\lambda=320 \mathrm{~nm}$ for $(-)-\mathrm{CMZ}$ in the absence or presence of different guests. The corresponding data for (+)-CMZ are presented in Figure S7 of the SI.

To investigate the protection provided by the different microenvironments, the triplet lifetimes were determined in the presence of increasing concentrations of oxygen. In all microheterogeneous systems, quenching by oxygen was slower than in homogeneous solution $\left(\mathrm{k}_{\mathrm{q}}=4.0 \times 10^{9} \mathrm{M}^{-1} \mathrm{~s}^{-1}\right)$; the effect was more remarkable inside glycoproteins $\left(\mathrm{k}_{\mathrm{q}}=9.0 \times 10^{7} \mathrm{M}^{-1} \mathrm{~s}^{-1}\right)$ than within CDXs $\left(\mathrm{k}_{\mathrm{q}}=1.9-2.3 \times 10^{9} \mathrm{M}^{-1} \mathrm{~s}^{-1}\right)$. 
However, the fact that the highest protection from photooxidation to CMZ-SONO was achieved inside SDS micelles (see above), where triplet quenching by oxygen was only slightly retarded $\left(\mathrm{k}_{\mathrm{q}}=3.1 \times 10^{9} \mathrm{M}^{-1} \mathrm{~s}^{-1}\right)$ does not support a reaction pathway involving ${ }^{3} \mathrm{CMZ}^{*}$ as the key intermediate. This is in good agreement with the assumption that CMZ-SONO arises from trapping of $\mathrm{CMZ}^{+\bullet}$ by oxygen. ${ }^{25}$ As a matter of fact, photoionization does occur in PBS solution (see above), whereas it is nearly negligible in SDS and AAGs.

\section{Conclusions}

The photooxidation of cyamemazine occurs through photoionization and subsequent trapping of the resulting radical cation by oxygen, eventually leading to the corresponding sulfoxide. This reaction is extremely sensitive to the medium and constitutes an appropriate probe for localization of the drug within a variety of microenvironments. The encapsulation process can be followed by fluorescence spectroscopy, through a clear hypsochromic shift, together with significantly enhanced quantum yields and lifetimes. Laser flash photolysis also reveals diagnostically important changes associated with encapsulation, specifically more selective generation of the triplet excited state, which turns out to be much longer-lived. Interestingly, as anticipated from the lipophilic nature of the employed microenvironments, photoionization to afford the radical cation is disfavored. Upon steady-state irradiation, formation of the sulfoxide can be easily monitored by fluorescence spectroscopy, by the progressive appearance of a characteristic emission band at shorter wavelengths. A good correlation is observed between the photophysical properties and the photochemical reactivity. Thus, the slowest photooxidation rate corresponds to cyamemazine in SDS micelles and $\alpha_{1}$-acid glycloproteins, where the drug is located in a more hydrophobic 
domain, hardly accessible from the aqueous medium, and photoionization is nearly negligible.

\section{Acknowledgments}

Financial support from the Spanish Government (CTQ2010-14882, BES-2011-043706, JCI-2010-06204) and from the Generalitat Valenciana (PROMETEOII/2013/005) is gratefully acknowledged.

\section{References}

${ }^{1}$ Feinberg, A. P. and S. H. Snuder (1975) Phenothiazine drugs: structure-activity relationships explained by a conformation that mimics dopamine. Proc. Nat. Acad. Sci. 72, 1899-1903.

${ }^{2}$ Jaszczyszyn, A., K. Gasiorowski, P. Swiatek, W. Malinka, K. Cieoelik-Boczula, J. Petrus and B. Czarnik-Matusewicz (2012) Chemical structure of phenothiazines and their biological activity. Pharmacol, Rep. 64, 16-23.

${ }^{3}$ Domínguez, J. N., S. López, J. Charris, L. Iarruso, G. Lobo, A. Semenow, J. E. P. Olson and J. Rosenthal (1997) Synthesis and antimalarial effects of phenothiazine inhibitors of a plasmodium falciparum cysteine protease. J. Med. Chem. 40, 2726-2732. ${ }^{4}$ Aaron, J. J., M. D. Gaye Seye, S. Trajkovska and N. Motohashi (2009) Bioactive phenothiazines and benzo[a]phenothiazines: spectroscopic studies and biological and biomedical properties and applications. Top Heterocycl. Chem. 16, 153-231.

${ }^{5}$ White, N. D. (2013) Drug-induced photosensitivity and the major culprits. Am. J. Lifestyle Med. 7, 189-191.

${ }^{6}$ Onoue, S., M. Kato, R. Inoue, Y. Seto and S. Yamada (2014) Photosafety screening of phenothiazine derivatives with combined use of photochemical and cassette-dosing pharmacokinetic data. Toxicol. Sci. 137, 469-477.

${ }^{7}$ Albini, A., E. Fasani, B. D. Glass, M. E. Brown and P. M. Drummond (1998) Photoreactivity versus activity of a selected class of phenothiazines: a comparative study, in drugs, Photochemistry and Photostability, A. Albini, E. Fasani Eds, Royal Society of Chemistry. 
$\overline{{ }^{8} \text { Elisei, F. L., L. Latterini, G. G. Aloisi, U. Mazzucato, G. Viola, G. Miolo, D. Vedaldi }}$ and F. Dall'Acqua (2002) Excited-state properties and in vitro phototoxicity studies of three phenothiazine derivatives. Photochem. Photobiol. 75, 11-21.

${ }^{9}$ García, C., L. Piñero, R. Oyola and R. Arce (2009) Photodegradation of 2-Chloro Substituted Phenothiazines in Alcohols, Photochem Photobiol. 85, 160-170.

${ }^{10}$ Ronzani, F., A. Trivella, E. Arzoumanian, S. Blanc, M. Sarakha, C. Richard, E. Oliveros and S. Lacombe (2013) Comparison of the photophysical properties of three phenothiazine derivatives: transient detection and singlet oxygen production. Photochem. Photobiol. Sci. 12, 2160-2169.

${ }^{11}$ Peters, T. (1995) All about albumins: biochemistry genetics and medical applications, Academic Press, San Diego.

12 Fournier T., N. Medjoubi-N and D. Porquet (2000) Alpha-1-acid glycoprotein. Biochim. Biophys. Acta 1482, 157-171.

${ }^{13}$ El-Gamal, S., U. Wollert and W. E. Müller (1983) Binding of several phenothiazine neuroleptics to a common binding site of alpha1-acid glycoprotein, Orosomucoid. $J$ Pharm Sci. 72, 202-205.

${ }^{14}$ Miyoshi, T., K. Sukimoto and M. Otagiri (1992) Investigation of the interaction mode of phenothiazine neuroleptics with $\alpha 1$-acid glycoprotein. J. Pharm. Pharmacol. 44, $28-33$.

15 Taheri, S., L. P. Cogswell, A. Gent and G. R. Strichartz (2003) Hydrophobic and ionic factors in the binding of local anesthetics to the major variant of human 1-acid glycoprotein. J. Pharm. Exp. Therap. 304, 71-80.

${ }^{16}$ Schill, G., I. W. Wainer and S. A. Barkan (1986) Chiral separations of cationic and anionic drugs on an $\alpha 1$-acid glycoprotein-bonded stationary phase (enantiopac ${ }^{\circledR}$ ): II. Influence of mobile phase additives and $\mathrm{pH}$ on chiral resolution and retention. J. Liq. Chrom. A 365, 73-88.

${ }^{17}$ Mischita, T., P. Franco and T. Zhang (2010) New approaches of LC-MS compatible method development on $\alpha 1$-acid glycoprotein-based stationary phase for resolution of enantiomers by HPLC. J. Sep. Sci. 33, 3627-3637.

${ }^{18}$ Hermansson, J. and A. Grahn (1995) Optimization of the separation of enantiomers of basic drugs retention mechanisms and dynamic modification of the chiral bonding properties on a $\alpha_{1}$-acid glycoprotein column. J. Chrom. A 694, 57-69. 
19 Lutka, A. (2000) Effect of cyclodextrin complexation on aqueous solubility and photostability of phenothiazine. Pharmazie 55, 120-123.

${ }^{20}$ Caetano, W. and T. Marcel (2000) Interaction of chlorpromazine and trifluoperazine with anionic sodium dodecyl sulfate (SDS) micelles: electronic absorption and fluorescence studies. J. Coll. Interf. Sci. 225, 69-81.

${ }^{21}$ Ghosh, H. N., A. V. Sapre, D. K. Palit and J. P. Mittal (1997) Picosecond flash photolysis studies on phenothiazine in organic and micellar solution. J. Phys. Chem. B 101, 2315-2320.

${ }^{22}$ Irie, T., M. Sunada, M. Otagiri and K. Uekama (1983) Protective mechanism of $\beta$ cyclodextrin for the hemolysis induced by phenothiazine neuroleptics in vitro. $J$. Pharmacobio-Dyn. 6, 408-414.

${ }^{23}$ Chankvetadze, B., I. Kartozia, N. Burjanadze, D. Bergenthal, H. Luflrnann and G. Blaschke (2001) Enantiosparation of chiral phenothiazine derivatives in capillary electrophoresis using cyclodextrin type chyral selectors. Chromatographia 53, 290-295.

${ }^{24}$ Cornilleau, V., A. Dompmartin, L. Michel, L. Verneuil and D. Leroy (2000) Photoscratch testing in systemic drug-induced photosensitivity. Photodermatol. Photoimmunol. Photomed. 16, 62-66.

${ }^{25}$ Morliere, P., F. Bosca, M. A. Miranda, J. V. Castell and R. Santus (2004) Primary photochemical processes of the phototoxic neuroleptic cyamemazine: a study by laser flash photolysis and steady-state irradiation. Photochem. Photobiol. 80, 535-541.

${ }^{26}$ Morliere, P., J. Haigel, K. Aissani, P. Filipe, J. N. Silva and R. Santus (2004) An insight into the mechanism of the phototoxic response induced by cyamemazine in cultured fibroblasts and keratinocytes. Photochem Photobiol. 79, 163-171. 